\title{
"هقارنة طرائق التقدير التقريبية لمعلمتي التوزيع اللوجستي"
}

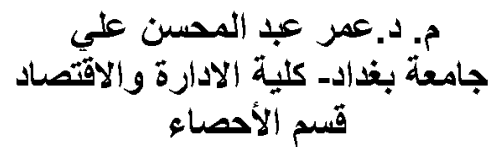

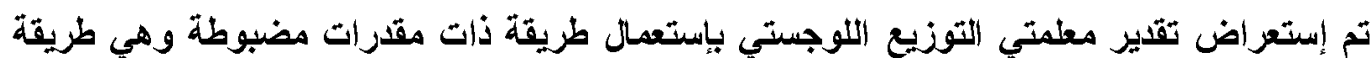

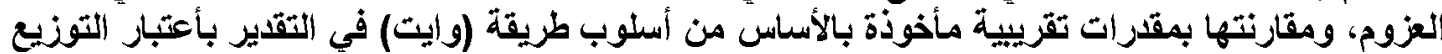

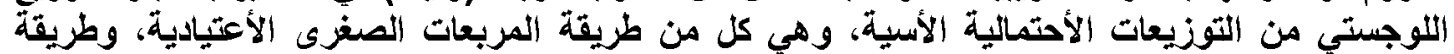

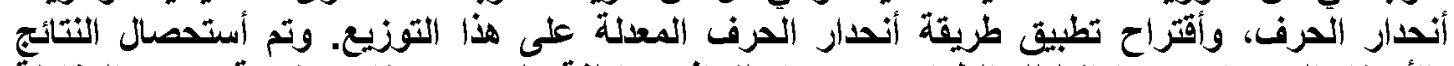

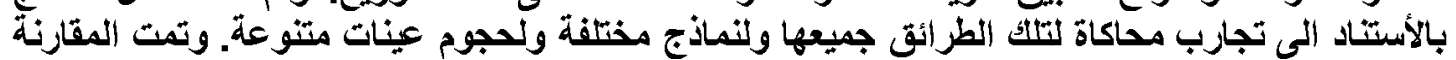
بالأستناد الى معياري متوسط مربع الخطأ ومتوسط الخئ الخطأ النسبي المطلق.

\section{$\underline{\text { Abstract }}$}

The goal beyond this Research is to review methods that used to estimate Logistic distribution parameters. An exact estimators method which is the Moment method, compared with other approximate estimators obtained essentially from White approach such as: OLS, Ridge, and Adjusted Ridge as a suggested one to be applied with this distribution. The Results of all those methods are based on Simulation experiment, with different models and variety of sample sizes. The comparison had been made with respect to two criteria: Mean Square Error (MSE) and Mean Absolute Percentage Error (MAPE).

1.1

يعد التوزيع اللوجستي من التوزيعات الحيوية في الأحصاء، وهو توزيع بارز من توزيعات التونية العائلة

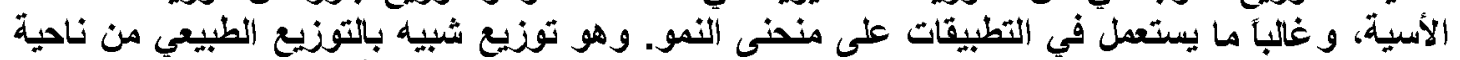

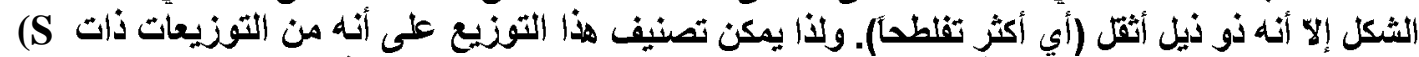
- Shaped)

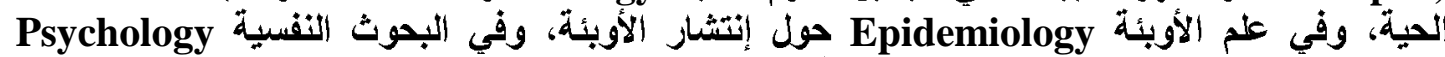

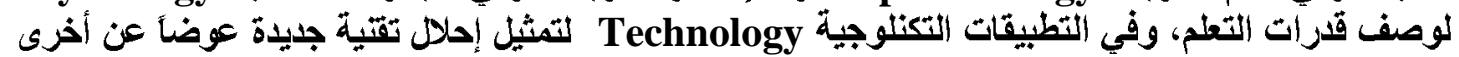

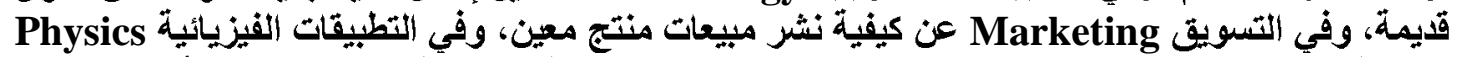

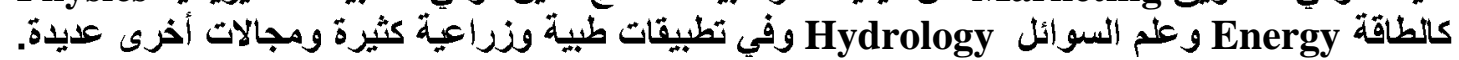

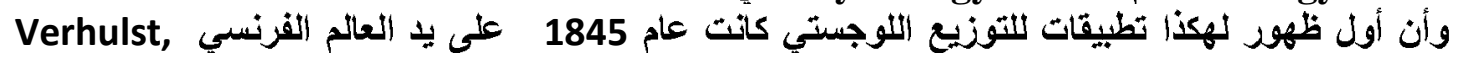

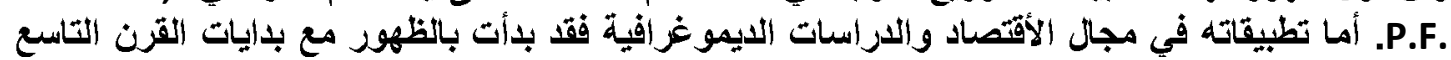




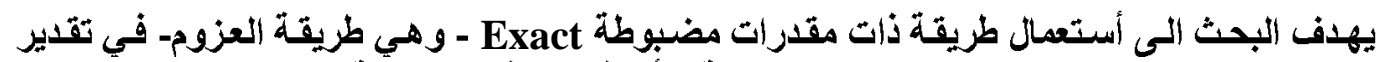

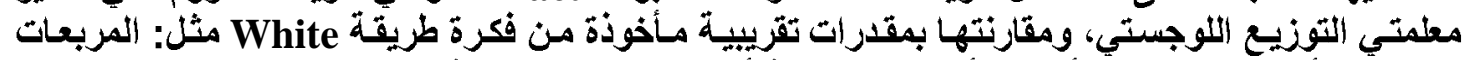

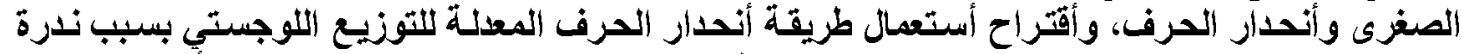

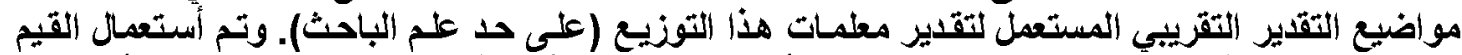

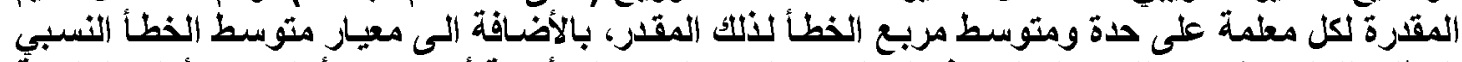

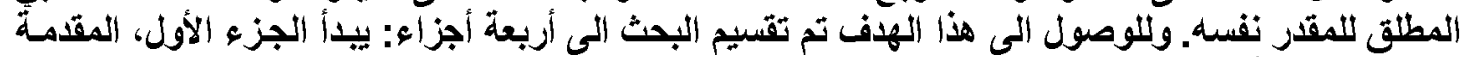

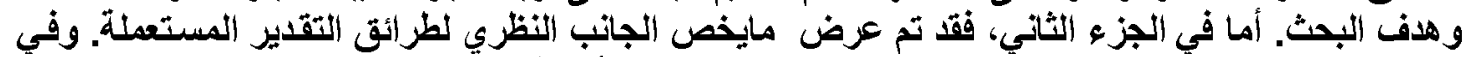

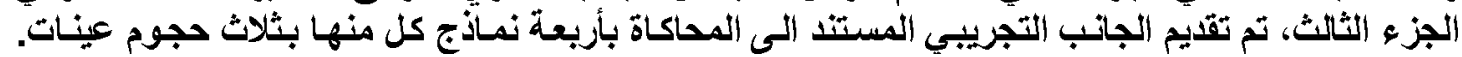

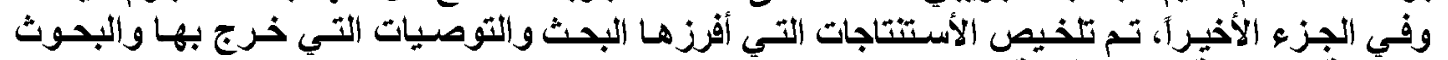

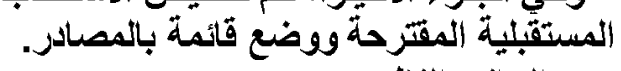

\section{Logistic Distribution}

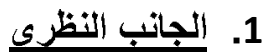

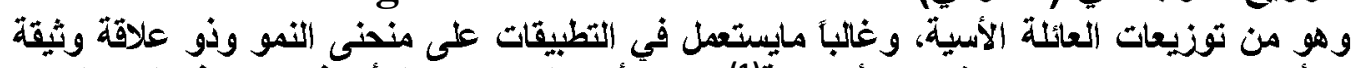

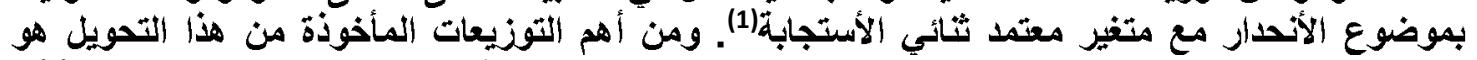

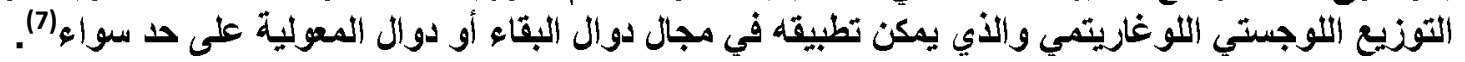

Characteristics of the Distribution

i) Probability Density Function (pdf)

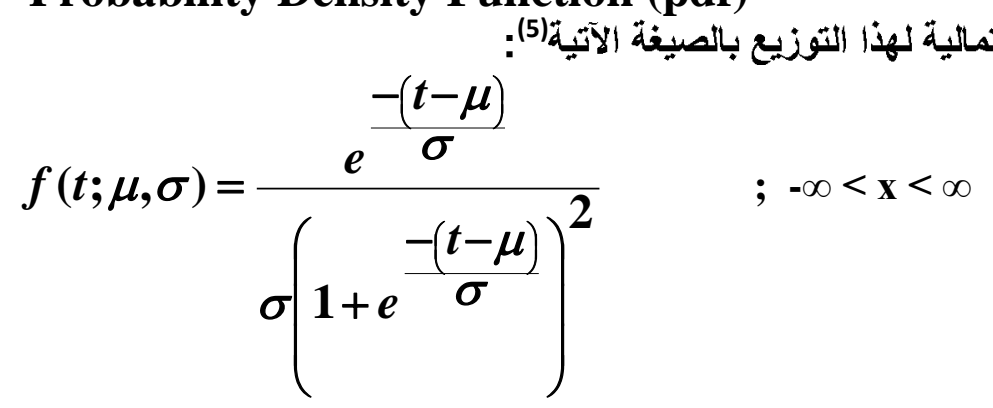

1.1.2

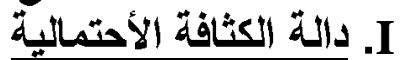

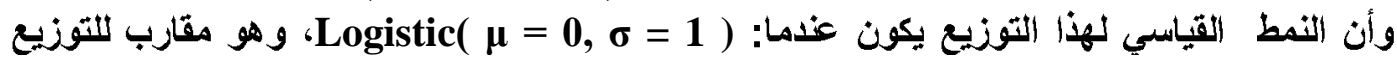

$$
\text { الطبيعى }
$$

Cumulative Distribution function (cdf)

$$
\begin{aligned}
& F(t ; \mu, \sigma)=\frac{1}{1+e^{\frac{-(t-\mu)}{\sigma}}} \quad ;-\infty<x<\infty \\
& f(t)=F(t)[1-F(t)]
\end{aligned}
$$

وتمثل بالصيغة الآتية|كمية:

ويمكن ملاحظة أن: 
ويتت Moment Generating Function (mgf)

$$
\begin{array}{r}
M(t)=e^{\mu t} \cdot \Gamma(1-\sigma t) \cdot \Gamma(1+\sigma t) \\
M(t)=e^{\mu t} \cdot B(1-\sigma t, 1+\sigma t)
\end{array}
$$

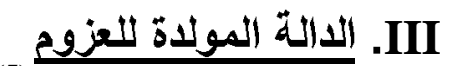
التعبير عنها بالصيغة الآتية:)(5):

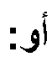

(1)

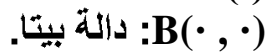

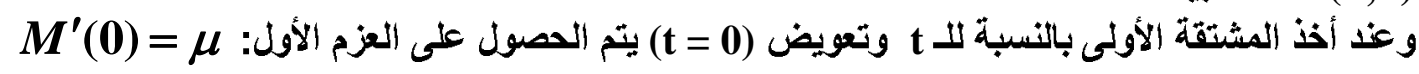

أما العزم الثاني فيتم الحصول عليه من أخذ المشتقة الثانية للصيغة (3) أعلاه بالنسبة للا t وتعويض (t=0): $M^{\prime \prime}(0)=\mu^{2}+\frac{\pi^{2} \sigma^{2}}{3}$

\section{Mean and Variance}

IV

$E(t)=\mu$ من ملاحظة المعادلة (4) أعلاه يتبين أن وسط التوزيع هو:

$\operatorname{Var}(t)=E\left(t^{2}\right)-[E(t)]^{2}$

أما تباين التوزيع فيمكن الحصول طليه بالأستناد الى المعائلة (4) و (5) أعلاه:

$\operatorname{Var}(t)=\frac{\pi^{2} \sigma^{2}}{3}$

Estimation Methods

Method of Moments (MOM)

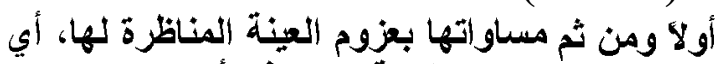

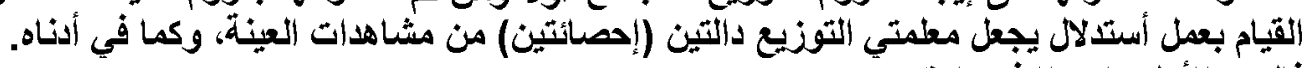

$M_{1}=E(t)=\mu$

$m_{1}=\bar{t}$

$\hat{\mu}=\bar{t}$

$M_{2}=\operatorname{Var}(t)+M_{1}^{2}$

$m_{2}=\frac{\sum_{i=1}^{n} t_{i}^{2}}{n}$

$\hat{\sigma}=\frac{\sqrt{3}}{\pi} S_{d}$ فالعزم الأول ماهو إلاذ عبارة عن: 
$S_{d}=\sqrt{\frac{\sum_{i=1}^{n} t_{i}^{2}}{n}-\left(\frac{\sum_{i=1}^{n} t_{i}}{n}\right)^{2}}$

Ordinary Least Squares (OLS) و طريقة المربعات الصغرى الأعتيادية

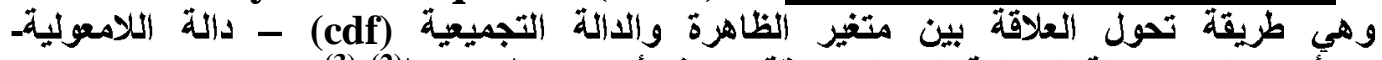

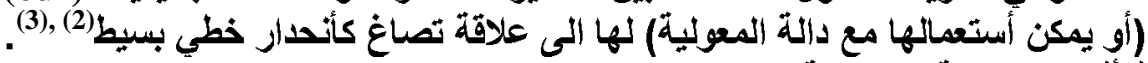

$$
\begin{aligned}
& u=\frac{1}{1+e^{\frac{-(t-\mu)}{\sigma}}} \\
& e^{\frac{-(t-\mu)}{\sigma}}=u^{-1}-1 \\
& \mathbf{u}=\mathbf{F}(\mathbf{t}) \\
& \text { وليكن: } \\
& \text { ليتم الحصول على: }
\end{aligned}
$$

وبأخذ لوغاريتم الطبيعي (In) لطرفي المعادلة أعلاه نحصل على:

$-\frac{t-\mu}{\sigma}=\ln \left(u^{-1}-1\right)$

$\ln \left(u_{i}^{-1}-1\right)=\frac{\mu}{\sigma}-\frac{1}{\sigma} t_{i}$

$$
Y_{i}=b_{0}+b_{1} X_{i}+e_{i}
$$

ويالنظر الى الصيغة (9) أعلاه كنموذج أندار خطي بسيط،

أذ أن: $X_{i}=t_{i}$ وغد أخذ التعويض بنظر الأعتبار يتم الحصول على:

$b_{0}=\frac{\mu}{\sigma}$

$b_{1}=\frac{-1}{\sigma}$

$Y_{i}=\ln \left(u^{-1}-1\right)$ 
$\hat{Y}_{i}=\hat{b}_{0}+\hat{b}_{1} X_{i}$

والغرض من هذا كله هو أجراء تقيير مطلمات الصيغة (10) أعلاه، وكما يأتي:

أن أساس طريقة المريعات الصغرى هو السعي الى تصنير مجموع مريعات الخطأ(2) (3):

$\sum_{i=1}^{n} e_{i}^{2}=\sum_{i=1}^{n}\left(Y_{i}-b_{0}-b_{1} X_{i}\right)^{2}$

وذلكك بأخذ المشتقة الجزئية للصيغة (13) بالنسبة للمطنتين $\hat{b}_{(o l s)}=\left[\begin{array}{l}\hat{b}_{0} \\ \hat{b}_{1}\end{array}\right]=\left(X^{\prime} X\right)^{-1} X^{\prime} Y$ القيم التقليرية لها

فتكون تقليرات معلتي النموذج اللوجستي الأحتمالي بلالة مقرات طريقة OLS لمعلتي الأحدار الخطي

$$
\begin{gathered}
\hat{\mu}_{(O L S)}=\frac{-\hat{b}_{0}(O L S)}{\hat{b}_{1(O L S)}} \\
\hat{\sigma}_{(O L S)}=\frac{-1}{\hat{b}_{1(O L S)}}
\end{gathered}
$$

Ridge Regression Method يأتي (4): هي من الطرائق التي تعول على أيجاد مقدرات الأندار بالأستثاد الى مصفوفة المعلومات X'X وكما $\hat{b}_{(\operatorname{Rig})}=\left[\begin{array}{l}\hat{b}_{0} \\ \hat{b}_{1}\end{array}\right]=\left(X^{\prime} X+k I\right)^{-1} X^{\prime} Y$

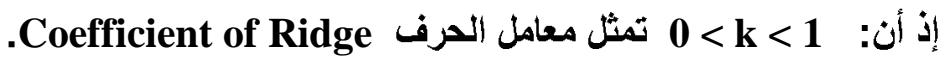

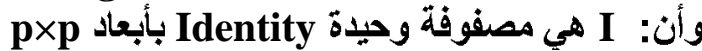

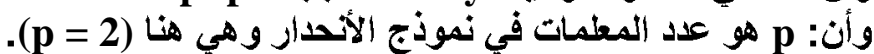

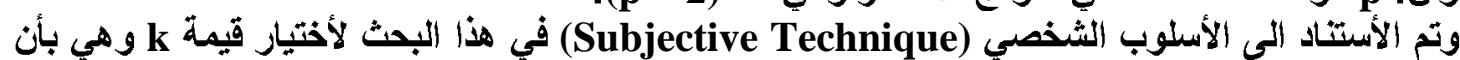

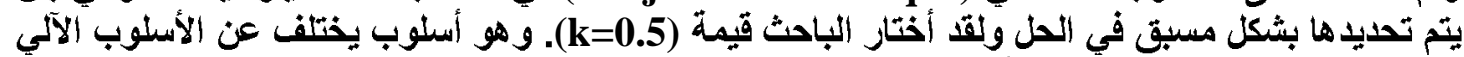

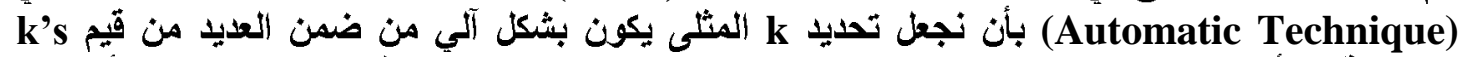

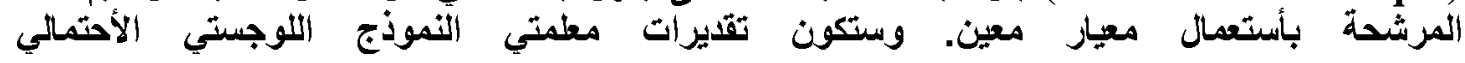
(Ridge بدلالة مقرات طريقي الأنحار الخطي بمثل ماجاء في المعائتين

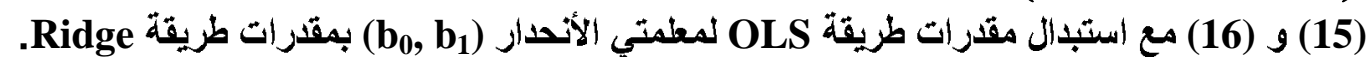




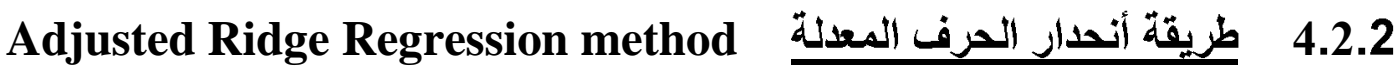

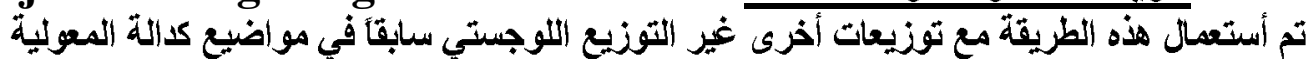

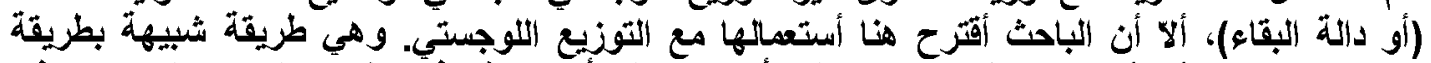

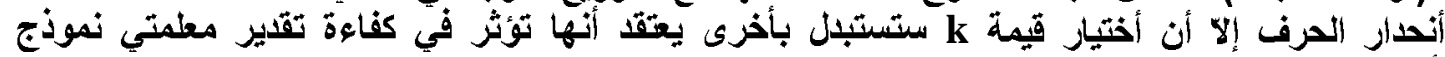
$\hat{\underline{b}}_{\text {(ARig. })}=\left[\begin{array}{l}\hat{b}_{0} \\ \hat{b}_{1}\end{array}\right]=\left(X^{\prime} X+k_{a d j} I\right)^{-1} X^{\prime} Y$

$k_{a d j}=\frac{p S_{O L S}}{\underline{\hat{b}}_{O L S} \underline{\hat{b}}_{\text {OLS }}}$

أذ أن:

$S_{o l s}=\frac{\sum_{i=1}^{n}\left(Y_{i}-\hat{b}_{0}-\hat{b}_{1} X_{i}\right)^{2}}{n-p}$

وأن:

وستكون تقيرات معلمتي النموذج اللوجستي الأحتمالمى $)$ (ARidg.) ARidge لمطلتي الأحدار الخطي بمثل ماجاء في المعالثتين (15) و (16) مع استبدال مقرات طريقة ARidge بمعثني الأنحدار (bo

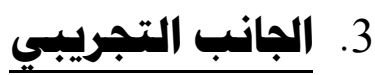

1.3

تم أعداد برنامجاً خاصاً بإستعمال لغة (Release 14)

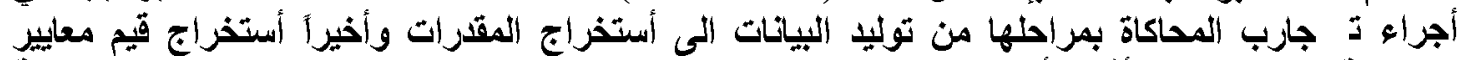

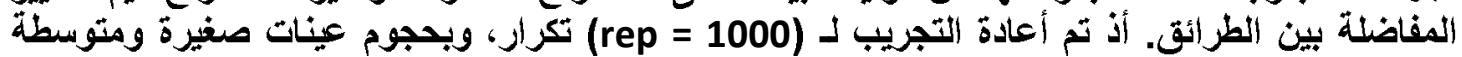
وكبيرة (n= 15, 30, 100) وللنماذج الآتية: النموذج الأول:

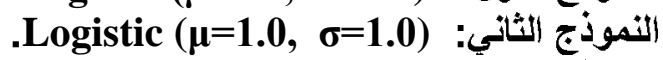
النموذج الثالث: النموذج الزابع: $t_{i}=\mu-\sigma \ln \left(u_{i}^{-1}-1\right)$ ويتم توليي بيانات التوزيع اللوجستي كما يأني:

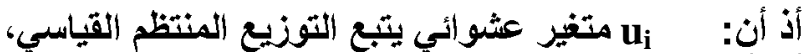
أي أن: 
Mean Squared Error (MSE)

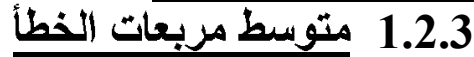
ويمثل معيار مقارنة تكون فيه ألَفضلية للقيمة الأصغر الأقرب الى الصفر، وكما في الصيغة: $M S E=\frac{\sum_{i=1}^{r e p}\left\{\theta_{i}-\hat{\theta}_{i}\right\}^{2}}{\text { rep }}$

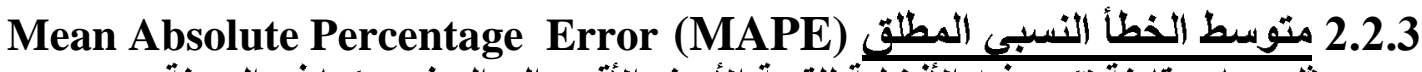
ويمثل معيار مقارنة تكون فيه الأقضلية للقيمة الأصغر الأقرب الى الصفر، وكما في الصيغة:

$$
\text { MAPE }=\frac{\sum_{i=1}^{r e p}\left|\theta_{i}-\hat{\theta}_{i}\right|}{\text { rep }}
$$

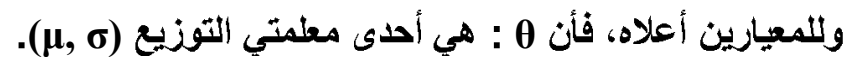

$$
\begin{aligned}
& \text { وأن rep: هو عدد التكرارات في تجرية المحاكاة. }
\end{aligned}
$$

3.3

تم الحصول على نتائج تقدير معلمتي التوزيع اللجستي وللنماذج وحجوم العينات المستعلة مع معياري

\begin{tabular}{|c|c|c|c|c|c|}
\hline \multirow{3}{*}{ Model } & & \multirow{2}{*}{\multicolumn{4}{|c|}{ Method }} \\
\hline & \multirow{2}{*}{ Sample size } & & & & \\
\hline & & MOM & OLS & Ridg. & ARidg \\
\hline \multirow{3}{*}{ I } & 15 & 1.0059 & 1.0031 & \begin{tabular}{|l|l|}
0.9684 \\
\end{tabular} & 0.9836 \\
\hline & 30 & 1.0056 & 1.0004 & 0.9834 & 0.9912 \\
\hline & 100 & 1.0010 & 0.9992 & 0.9967 & 1.0017 \\
\hline \multirow{3}{*}{ II } & 15 & 1.0118 & 0.9383 & \begin{tabular}{|l|l}
0.9717 \\
\end{tabular} & 1.0063 \\
\hline & 30 & 1.0113 & 0.9663 & 0.9839 & 1.0008 \\
\hline & 100 & 1.0012 & 0.9933 & \begin{tabular}{|l|}
0.9985 \\
\end{tabular} & 1.0035 \\
\hline \multirow{3}{*}{ III } & 15 & 1.0177 & 0.8815 & \begin{tabular}{|l|l|}
0.9749 \\
\end{tabular} & 1.0094 \\
\hline & 30 & 1.016 & 0.9300 & 0.9843 & 1.0012 \\
\hline & 100 & 1.0031 & 0.9829 & \begin{tabular}{|l}
1.0002 \\
\end{tabular} & 1.0053 \\
\hline \multirow{3}{*}{ IV } & 15 & 1.0236 & 0.8264 & \begin{tabular}{|l|l}
0.9781 \\
\end{tabular} & 1.0126 \\
\hline & 30 & 1.0226 & 0.8882 & \begin{tabular}{|l|}
0.9848 \\
\end{tabular} & 1.0017 \\
\hline & 100 & 1.0041 & 0.9684 & $\begin{array}{l}1.0020 \\
\end{array}$ & 1.0070 \\
\hline
\end{tabular}
المقارنة MSE و عAPE وكما في الجداول أنداه. 
جلول (2) متوسط مربعات الخطأ MSE لتقديرات معلمة الموقع

\begin{tabular}{|c|c|l|l|l|l|l|}
\hline \multirow{2}{*}{ Model } & \multirow{2}{*}{ Sample size } & \multicolumn{4}{|c|}{ Method } & \multirow{2}{*}{ Best } \\
\cline { 2 - 6 } & MOM & OLS & Ridg. & ARidg. & \\
\hline \multirow{4}{*}{ I } & $\mathbf{1 5}$ & $\mathbf{0 . 0 5 9 0 1}$ & $\mathbf{0 . 0 2 0 1 4}$ & $\mathbf{0 . 0 2 5 0 1 7}$ & $\mathbf{0 . 0 2 4 1 3}$ & OLS \\
\cline { 2 - 7 } & $\mathbf{3 0}$ & $\mathbf{0 . 0 2 7 8 4}$ & $\mathbf{0 . 0 0 9 7 7}$ & $\mathbf{0 . 0 1 0 7 0}$ & $\mathbf{0 . 0 1 0 4}$ & OLS \\
\cline { 2 - 7 } & $\mathbf{1 0 0}$ & $\mathbf{0 . 0 0 8 5}$ & $\mathbf{0 . 0 0 2 6}$ & $\mathbf{0 . 0 0 2 7}$ & $\mathbf{0 . 0 0 2 6}$ & OLS \\
\hline \multirow{3}{*}{ II } & $\mathbf{1 5}$ & $\mathbf{0 . 2 3 6 0}$ & $\mathbf{0 . 0 9 3 1 2}$ & $\mathbf{0 . 0 8 0 5 6}$ & $\mathbf{0 . 0 8 0 1 9}$ & ARidg. \\
\cline { 2 - 7 } & $\mathbf{3 0}$ & $\mathbf{0 . 1 1 1 3}$ & $\mathbf{0 . 0 4 2 3 5}$ & $\mathbf{0 . 0 3 9 0 8}$ & $\mathbf{0 . 0 3 8 9 4}$ & ARidg. \\
\cline { 2 - 7 } & $\mathbf{1 0 0}$ & $\mathbf{0 . 0 3 4 2}$ & $\mathbf{0 . 0 1 0 8 3}$ & $\mathbf{0 . 0 1 0 6 5}$ & $\mathbf{0 . 0 1 0 6 1}$ & ARidg. \\
\hline \multirow{3}{*}{ III } & $\mathbf{1 5}$ & $\mathbf{0 . 5 3 1 1}$ & $\mathbf{0 . 1 9 3 9}$ & $\mathbf{0 . 1 8 1 2}$ & $\mathbf{0 . 1 7 3 3}$ & ARidg. \\
\cline { 2 - 7 } & $\mathbf{3 0}$ & $\mathbf{0 . 2 5 0 6}$ & $\mathbf{0 . 0 9 5 0}$ & $\mathbf{0 . 0 8 7 9}$ & $\mathbf{0 . 0 8 6 1}$ & ARidg. \\
\cline { 2 - 7 } & $\mathbf{1 0 0}$ & $\mathbf{0 . 0 7 7 1}$ & $\mathbf{0 . 0 2 4 5}$ & $\mathbf{0 . 0 2 3 7}$ & $\mathbf{0 . 0 2 3 9}$ & ARidg. \\
\hline \multirow{3}{*}{ IV } & $\mathbf{1 5}$ & $\mathbf{0 . 9 4 4 3}$ & $\mathbf{0 . 3 2 2 2}$ & $\mathbf{0 . 3 1 1 4}$ & $\mathbf{0 . 3 0 4 0}$ & ARidg. \\
\cline { 2 - 7 } & $\mathbf{3 0}$ & $\mathbf{0 . 4 4 5 5}$ & $\mathbf{0 . 1 5 6 3}$ & $\mathbf{0 . 1 6 4 8}$ & $\mathbf{0 . 1 5 2 1}$ & ARidg. \\
\cline { 2 - 6 } & $\mathbf{1 0 0}$ & $\mathbf{0 . 1 3 7 1}$ & $\mathbf{0 . 0 4 2 6}$ & $\mathbf{0 . 0 4 2 2}$ & $\mathbf{0 . 0 4 3 8}$ & Ridg. \\
\hline
\end{tabular}

جدول (3) متوسط الخطأ النسبي المطلق MAPE لتقديرات معلمة الموقع Med

\begin{tabular}{|c|c|c|c|c|c|c|}
\hline \multirow{2}{*}{ Model } & \multirow{2}{*}{ Sample size } & \multicolumn{4}{|c|}{ Method } & \multirow{2}{*}{ Best } \\
\cline { 2 - 6 } & MOM & OLS & Ridg. & ARidg. & \\
\hline \multirow{4}{*}{ I } & $\mathbf{1 5}$ & $\mathbf{0 . 1 3 8 0}$ & $\mathbf{0 . 1 1 9 7}$ & $\mathbf{0 . 1 2 3 2}$ & $\mathbf{0 . 1 1 1 6}$ & ARidg. \\
\cline { 2 - 6 } & $\mathbf{3 0}$ & $\mathbf{0 . 1 0 9 9}$ & $\mathbf{0 . 0 7 7 3}$ & $\mathbf{0 . 0 8 1 2}$ & $\mathbf{0 . 0 8 0 0}$ & OLS \\
\cline { 2 - 6 } II & $\mathbf{1 0 0}$ & $\mathbf{0 . 0 5 0 1}$ & $\mathbf{0 . 0 4 1 5}$ & $\mathbf{0 . 0 4 1 6}$ & $\mathbf{0 . 0 4 1 2}$ & ARidg. \\
\cline { 2 - 7 } & $\mathbf{1 5}$ & $\mathbf{0 . 2 4 4 4}$ & $\mathbf{0 . 2 3 7 9}$ & $\mathbf{0 . 2 2 1 9}$ & $\mathbf{0 . 2 2 3 2}$ & Ridg. \\
\cline { 2 - 6 } & $\mathbf{3 0}$ & $\mathbf{0 . 1 8 5 2}$ & $\mathbf{0 . 1 5 4 6}$ & $\mathbf{0 . 1 5 4 6}$ & $\mathbf{0 . 1 6 1 5}$ & OLS \\
\hline \multirow{3}{*}{ III } & $\mathbf{1 0 0}$ & $\mathbf{0 . 0 8 1 1}$ & $\mathbf{0 . 0 8 3 2}$ & $\mathbf{0 . 0 8 2 3}$ & $\mathbf{0 . 0 8 2 4}$ & MOM \\
\cline { 2 - 6 } & $\mathbf{1 5}$ & $\mathbf{0 . 3 6 1 1}$ & $\mathbf{0 . 3 4 9 4}$ & $\mathbf{0 . 3 2 7 0}$ & $\mathbf{0 . 3 3 4 9}$ & Ridg. \\
\cline { 2 - 6 } & $\mathbf{1 0 0}$ & $\mathbf{0 . 2 7 0 0}$ & $\mathbf{0 . 2 4 3 7}$ & $\mathbf{0 . 2 2 9 8}$ & $\mathbf{0 . 2 3 2 0}$ & Ridg. \\
\hline \multirow{3}{*}{ IV } & $\mathbf{0 . 1 3 8 9}$ & $\mathbf{0 . 1 2 5 3}$ & $\mathbf{0 . 1 2 3 2}$ & $\mathbf{0 . 1 2 3 7}$ & Ridg. \\
\cline { 2 - 6 } & $\mathbf{3 0}$ & $\mathbf{0 . 4 9 2 3}$ & $\mathbf{0 . 4 5 0 0}$ & $\mathbf{0 . 4 4 6 5}$ & $\mathbf{0 . 4 3 3 4}$ & ARidg. \\
\cline { 2 - 6 } & $\mathbf{1 0 0}$ & $\mathbf{0 . 1 7 4 2}$ & $\mathbf{0 . 1 6 7 9}$ & $\mathbf{0 . 1 6 4 9}$ & $\mathbf{0 . 1 6 4 2}$ & ARidg. \\
\hline
\end{tabular}

يلاحظ من نتائج الجدول (2) أعلاه تفوق طريقة OLS على باقي الطرائق لنتائج النموذج الأول ولجميع

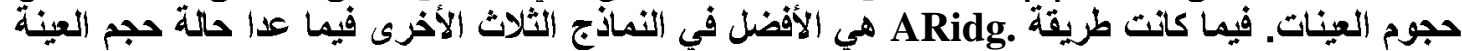

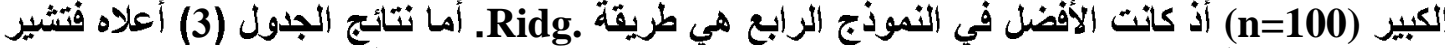
اللى تفوق طريقة OLS على باقي الطرائق لنتائج جميع النماذج ولحجم عينة (n=30) فقط. فيما كاتت طريقة . Ridg هي الأفضل للنموذج الثالث ولجميع حجوم العينات. 
جدول (4) تقديرات معلمة القياس

\begin{tabular}{|c|c|c|c|c|c|}
\hline \multirow{2}{*}{ Model } & \multirow{2}{*}{ Sample size } & \multicolumn{4}{|c|}{ Method } \\
\hline & & MOM & OLS & Ridg. & ARidg. \\
\hline \multirow{3}{*}{ I } & 15 & 0.4737 & 0.5427 & 0.5653 & 0.5111 \\
\hline & 30 & 0.4887 & 0.5177 & 0.5282 & 0.5052 \\
\hline & 100 & 0.4964 & 0.5050 & 0.5081 & 0.5018 \\
\hline \multirow{3}{*}{ II } & 15 & 0.9475 & 1.0818 & 1.0494 & 1.0223 \\
\hline & 30 & 1.0104 & 0.9775 & 1.0219 & 1.0351 \\
\hline & 100 & 0.9928 & 1.0100 & 1.0067 & 1.0036 \\
\hline \multirow{3}{*}{ III } & 15 & 1.4212 & 1.6155 & 1.5514 & 1.5334 \\
\hline & 30 & 1.4662 & 1.5516 & 1.5233 & 1.5156 \\
\hline & 100 & 1.4893 & 1.5150 & 1.5075 & 1.5054 \\
\hline \multirow{3}{*}{ IV } & 15 & 1.8950 & 2.1443 & 2.0580 & 2.0446 \\
\hline & 30 & 1.9550 & 2.0668 & 2.0266 & 2.0208 \\
\hline & 100 & 1.9857 & 2.0072 & 2.0199 & 2.0080 \\
\hline
\end{tabular}

جدول (5) متوسط مريعات الخطأ MSE لتقيرات معلمة القياس

\begin{tabular}{|c|c|c|c|c|c|c|}
\hline \multirow{2}{*}{ Model } & \multirow{2}{*}{ Sample size } & \multicolumn{4}{|c|}{ Method } & \multirow{2}{*}{ Best } \\
\hline & & MOM & OLS & Ridg. & ARidg. & \\
\hline \multirow{3}{*}{ I } & 15 & 0.0133 & 0.0160 & 0.0159 & 0.0086 & ARidg. \\
\hline & 30 & 0.0062 & 0.0045 & 0.0047 & 0.0034 & ARidg. \\
\hline & 100 & 0.0020 & 0.0008 & 0.0008 & 0.0007 & ARidg. \\
\hline \multirow{3}{*}{ II } & 15 & 0.0535 & 0.0601 & $\begin{array}{ll}0.03855 \\
\end{array}$ & $\begin{array}{l}0.0344 \\
\end{array}$ & ARidg. \\
\hline & 30 & 0.0248 & 0.0141 & 0.0146 & 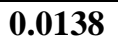 & ARidg. \\
\hline & 100 & 0.0030 & 0.0033 & 0.0032 & 0.0031 & MOM \\
\hline \multirow{3}{*}{ III } & 15 & 0.1204 & 0.1257 & 0.07743 & 0.0806 & Ridg. \\
\hline & 30 & 0.0558 & 0.0400 & 0.0310 & 0.0318 & Ridg. \\
\hline & 100 & 0.0180 & 0.0075 & 0.0070 & 0.0071 & Ridg. \\
\hline \multirow{3}{*}{ IV } & 15 & 0.21409 & 0.2097 & $\begin{array}{ll}0.1401 \\
\end{array}$ & 0.1376 & ARidg. \\
\hline & 30 & 0.09923 & 0.0698 & 0.0559 & 0.0552 & ARidg. \\
\hline & 100 & 0.03212 & 0.0126 & 0.0134 & 0.0130 & OLS \\
\hline
\end{tabular}




\begin{tabular}{|c|c|c|c|c|c|c|}
\hline \multirow{2}{*}{ Model } & \multirow{2}{*}{ Sample size } & \multicolumn{4}{|c|}{ Method } & \multirow{2}{*}{ Best } \\
\hline & & MOM & OLS & Ridg. & ARidg. & \\
\hline \multirow{3}{*}{ I } & 15 & 0.1898 & 0.1673 & 0.1747 & 0.1354 & ARidg. \\
\hline & 30 & 0.1023 & 0.0958 & 0.0986 & 0.0871 & ARidg. \\
\hline & 100 & 0.4800 & 0.0464 & 0.0470 & 0.0451 & ARidg. \\
\hline \multirow{3}{*}{ II } & 15 & 0.1505 & 0.1644 & 0.1403 & 0.1354 & ARidg. \\
\hline & 30 & 0.0997 & 0.0956 & 0.0885 & 0.0871 & ARidg. \\
\hline & 100 & 0.4498 & 0.0464 & 0.0454 & 0.0451 & MOM \\
\hline \multirow{3}{*}{ III } & 15 & 0.1411 & 0.1606 & 0.1354 & 0.1368 & Ridg. \\
\hline & 30 & 0.1064 & 0.0953 & 0.0871 & 0.0876 & Ridg. \\
\hline & 100 & 0.4676 & 0.0463 & 0.0451 & 0.0452 & Ridg. \\
\hline \multirow{3}{*}{ IV } & 15 & 0.1613 & 0.1570 & 0.1359 & 0.1354 & ARidg. \\
\hline & 30 & 0.0987 & 0.0948 & 0.0874 & 0.0871 & ARidg. \\
\hline & 100 & 0.0513 & 0.0450 & 0.0451 & 0.0463 & OLS \\
\hline
\end{tabular}

أفرزت نتاثج الجلولين (5) و (6) أفضلية واضحة لطريقة .ARidg على ماسواها من الطرائق، فيما

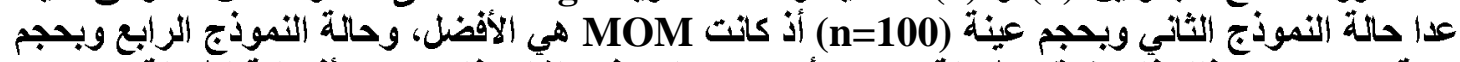

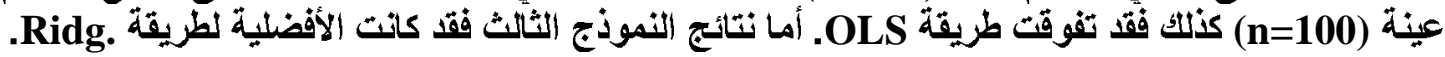

4. 1.4 الأستنتاجات والتوصيات (الأستتتاجات

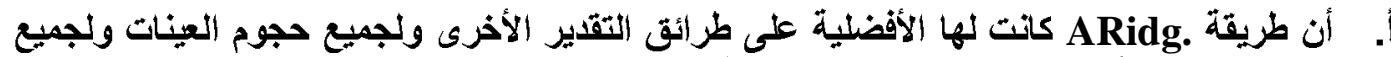

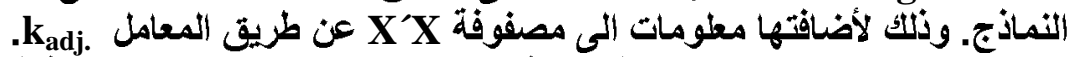

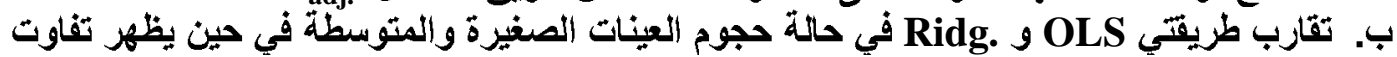

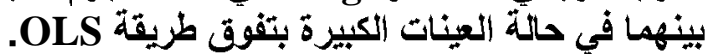
ج. تخاقص قَيم معياري المقارنة MSE و MAPE مع زيادة حجم العينة ولجميع النماذج ولجميع الطر ائقى. د. أظهرت نتائج النموذج الرابع عموماً أفضلية على النماذج الأخرى. 2.4

يوصى الباحث بأستعمال طريقة .ARidg لما لها من كفاءة عالئة متأتية من المعلومات الأضافية التي

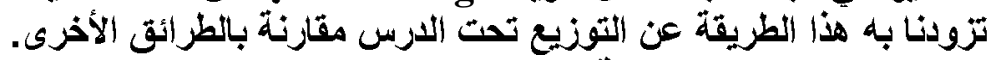
3.4

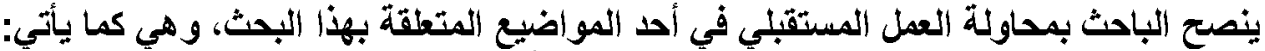

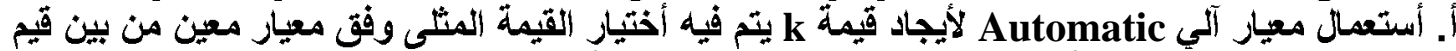

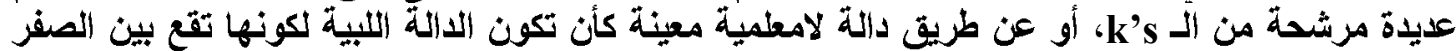

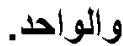

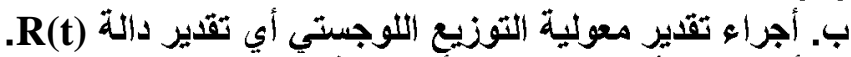

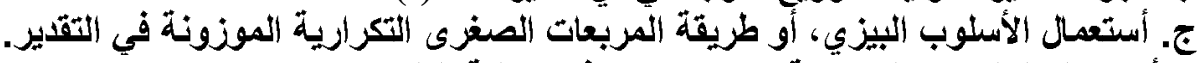
د. أستعمال المقاييس التجزيئية Quantiles في عملية التقدير. 


\section{$\underline{\text { References }}$}

1. Augustin, Thomas; (2005); "An Approach to Combine the Logistic Threshold Model of Psychophysics with Bradley - Terry - Luce Models of Choice Theory"; Journal of Mathematical Psychology Vol. 49, pp. 70-79.

2. Bickel, P.J. and Doksum, K.A.; (1977); "Mathematical Statistics: Basic Ideas and Selected Topics"; Holden-Day, Inc., San Francisco.

3. Feras, S.M. and Sharad, D.G.; (2009); "Ridge Regression Estimator: Combining Unbiased Ridge Regression Methods of Estimation"; Surveys in Mathematics an its Applications; Vol.

4. Ebeling, C.E.; (1997); "An Introduction to Reliability and Maintainability Engineering"; McGraw - Hill companies, New-York.

5. Johnson, Norman L. and Samuel Kotz; (1970); "Continuous Univariate Distributions - 2"; New York: Houghton Mifflin.

6. Mahdi, Smail and Cenac, Myrtene; (2006); "Estimating and Assessing the Parameters of the Logistic and Rayleigh Distributions from Three Methods of Estimation"; Caribb J. Math. Comput.

Sci.; Vol. 13, pp. 25 - 34.

7. Rao, G. S.; Kantam, R.; (2010); “ Estimation of Reliability in Multicomponent Stressstrength Model: Log-Logistic Distribution"; Journal of Applied Statistical Analysis, Vol. 3, No.2, pp. (75 - 84). 